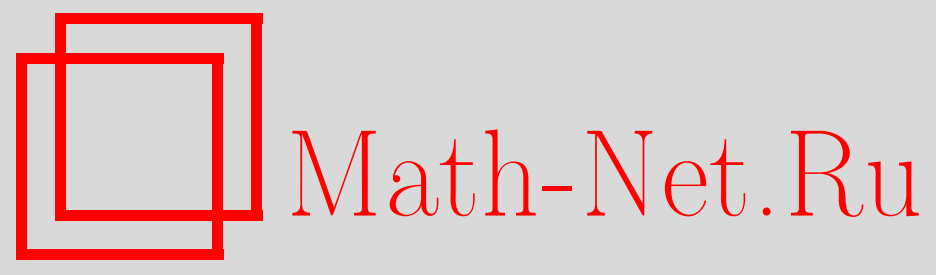

В. И. Юдович, Л. Г. Куракин, Ответвление предельного цикла от подмногообразия равновесий в системе с мультикосимметрией, Матем. заметки, 1999, том 66, выпуск $2,317-320$

DOI: https://doi.org/10.4213/mzm1172

Использование Общероссийского математического портала Math-Net.Ru подразумевает, что вы прочитали и согласны с пользовательским соглашением http://www. mathnet.ru/rus/agreement

Параметры загрузки:

IP : 54.237 .59 .107

26 апреля 2023 г., 18:04:48

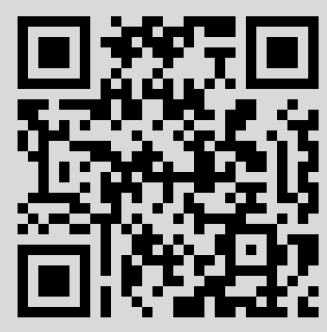




\section{ОТВЕТВЛЕНИЕ ПРЕДЕЛЬНОГО ЦИКЛА ОТ ПОДМНОГООБРАЗИЯ РАВНОВЕСИЙ В СИСТЕМЕ С МУЛЬТИКОСИММЕТРИЕЙ}

\section{Л.Г. Куракин, В.И. Юдович}

Для динамической системы, допускающей $k$ независимых косимметрий, типично существование $k$-мерных подмногообразий, заполненных равновесиями. В работах [1]-[3] в случае одной косимметрии были указаны новые эффекты, связанные с бифуркацией ответвления предельного цикла от семейства равновесий (запаздывание по параметру и возможность сверхкритических неустойчивых автоколебаний). Здесь эти результаты распространены на случай любого $k$. При $k>1$ приходится иметь дело с новым явлением - бифуркацией областей устойчивости на семействе равновесий. Применяются оба основных подхода к задаче возникновения автоколебаний: метод Ляпунова-Шмидта [4] и метод центрального многообразия [5]. Поскольку речь идет о локальной бифуркации, достаточно рассмотреть уравнение на линейном (гильбертовом) пространстве, отождествляя при этом векторы и ковекторы, а косимметрии (пфаффовы формы, аннулирующие данное векторное поле) с векторными полями. Подробно результаты этой заметки изложены в [6], [7].

1. Метод Ляпунова-Шмидта. Рассмотрим в гильбертовом пространстве $H$ обыкновенное дифференциальное уравнение

$$
\dot{\theta}=F(\theta, \lambda)
$$

с вещественным параметром $\lambda \in \mathbb{R}$. Здесь $F: H \times \mathbb{R} \rightarrow H$ - аналитический оператор, допускающий зависящую от $\lambda$ мульикосимметрию $\mathscr{L}:(\theta, \lambda) \rightarrow\left(L_{1}(\theta, \lambda), L_{2}(\theta, \lambda), \ldots, L_{k}(\theta, \lambda)\right)$, где $L_{i}: H \times \mathbb{R} \rightarrow H(i=1,2, \ldots, k)$ - известные операторы. Это означает [8], что для каждого отображения $L_{i}$ при всех $\theta \in H, \lambda \in \mathbb{R}$

$$
\left(F(\theta, \lambda), L_{i}(\theta, \lambda)\right)=0
$$

Пусть уравнение (1) при некотором $\lambda=\lambda_{0}$ имеет равновесие $\theta_{0}: F\left(\theta_{0}, \lambda_{0}\right)=0$. Тогда векторы $L_{0 i}=L_{i}\left(\theta_{0}, \lambda_{0}\right)(i=1, \ldots, k)$ принадлежат ядру оператора $A_{0}^{*}$, сопряженного к производной $A_{0}=F_{\theta}^{\prime}\left(\theta_{0}, \lambda_{0}\right)$ [8]. Таким образом, при условии линейной независимости системы $\left\{L_{01}, \ldots, L_{0 k}\right\}$ нуль является собственным значением оператора $A_{0}^{*}$ кратности $\geqslant k$.

Примем следующие гипотезы:

Н1) мультикосимметрия $\mathscr{L}$ непрерывна;

Н2) $\theta_{0}$ - регулярная точка мультикосимметрии $\mathscr{L}$, т.е. векторы $L_{0 i}(i=1, \ldots, k)$ образуют линейно независимую систему; ее линейную оболочку обозначим через $Y_{0}$;

Н3) выполнено условие минимальности вырохсдения: ядро $\operatorname{ker} A_{0}=Y_{0}$, так что $\operatorname{dim} \operatorname{ker} A_{0}=k$;

H4) пересечение спектра $\sigma\left(A_{0}\right)$ оператора $A_{0}$ с мнимой осью состоит из $k$-кратного нулевого и пары чисто мнимых $\pm i \omega_{0}, \omega_{0}>0$, простых собственных значений;

Н5) точки $0, \pm i \omega_{0}$ - полюсы оператора $A_{0}$, так что они являются собственными значениями как оператора $A_{0}$, так и его сопряженного $A_{0}^{*}$.

Работа выполнена при поддержке Российского фонда фундаментальных исследований, грант № 96-01-01791, и межвузовской программы "Университеты России - фундаментальные исследования", грант № 4087.

(С) Л.Г. КурАКин, В.И. Юдович

1999 
Пусть $\left\{\gamma_{1}, \gamma_{2}, \ldots, \gamma_{k}\right\}$-биортогональная система к набору функционалов $\left\{L_{01}, L_{02}, \ldots, L_{0 k}\right\}$; $\varphi, \Phi$ - собственные векторы операторов $A_{0}, A_{0}^{*}$ соответственно, так что $A_{0} \gamma_{j}=0,\left(\gamma_{j}, L_{0 l}\right)=\delta_{j l}$ $(j, l=1, \ldots, k), A_{0} \varphi=i \omega_{0} \varphi, A_{0}^{*} \Phi=-i \omega_{0} \Phi,(\varphi, \Phi)=1$.

Введем обозначения: $\widehat{H}=L_{2}\left(S^{1}, H\right)$ - гильбертово пространство вектор-функций на окружности $S^{1}$ со значениями в $H, \tau$ - угловая переменная на окружности, а $\widehat{H}^{0}$ - подпространство в $\widehat{H}$, состоящее из вектор-функций вида $u(\tau)=\alpha \varphi e^{i \tau}+\alpha^{*} \varphi^{*} e^{-i \tau}+\beta_{1} \gamma_{1}+\cdots+\beta_{k} \gamma_{k}$, где $\alpha \in \mathbb{C}$, $\beta_{j} \in \mathbb{R}$ - произвольные постоянные. Определим проектор $\Pi: \widehat{H} \rightarrow \widehat{H}^{0}$ следующим образом:

$$
(\Pi f)(\tau)=2 \operatorname{Re}\left[\left\langle(f, \Phi) e^{-i \tau}\right\rangle e^{i \tau} \varphi\right]+\sum_{j=1}^{k}\left(\langle f\rangle, L_{0 j}\right) \gamma_{j}, \quad\langle f\rangle \stackrel{\text { def }}{=} \frac{1}{2 \pi} \int_{0}^{2 \pi} f(\tau) d t
$$

Обозначим через $\widehat{H}^{\prime}$ дополнительное подпространство, а через $\Pi^{\prime}$ дополнительный проектор, так что $\widehat{H}=\widehat{H}^{0} \oplus \widehat{H}^{\prime}, \Pi^{\prime}=I-\Pi$.

Введем оператор $T: T u=\omega_{0} d u / d \tau-A_{0} u$, где $\tau=\omega_{0} t-$ новое время. Его сужение $T_{1}$ на $\widehat{H}^{\prime}-$ обратимый оператор.

Пусть $\mathscr{A}=\left(a_{i j}\right)_{i, j=0}^{k}$ - матрица, задаваемая выражениями

$$
\begin{aligned}
& a_{0 j}=\operatorname{Re} g_{j}, \quad g_{0}=\left\langle\left(F_{0}^{\prime \prime}\left(\psi, v_{2}\right)+\frac{1}{6} F_{0}^{\prime \prime \prime} \psi^{3}, \Phi\right) e^{-i \tau}\right\rangle, \quad g_{l}=\left(F_{0}^{\prime \prime}\left(\varphi, \gamma_{l}\right), \Phi\right), \\
& a_{l 0}=\left(\left\langle F_{0}^{\prime \prime}\left(\psi, v_{1}\right)+\frac{1}{2} F_{0}^{\prime \prime} v_{2}^{2}+\frac{1}{2} F_{0}^{\prime \prime \prime}\left(\psi, \psi, v_{2}\right)+\frac{1}{24} F_{0}^{\prime \prime \prime \prime} \psi^{4}\right\rangle, L_{0 l}\right), \\
& a_{l r}=\left(\left\langle F_{0}^{\prime \prime}\left(\psi, v_{1 r}\right)+F_{0}^{\prime \prime}\left(\gamma_{r}, v_{2}\right)+\frac{1}{2} F_{0}^{\prime \prime \prime}\left(\psi, \psi, \gamma_{r}\right)\right\rangle, L_{0 l}\right),
\end{aligned}
$$

где $l, r=1, \ldots, k$, функция $\psi(\tau)=\varphi e^{i \tau}+\varphi^{*} e^{-i \tau}$, a $v_{1}, v_{2}, v_{1 r} \in \widehat{H}^{\prime}$ :

$$
v_{1}=T_{1}^{-1} \Pi^{\prime}\left[\frac{1}{6} F_{0}^{\prime \prime \prime} \psi^{3}+F_{0}^{\prime \prime}\left(\psi, v_{2}\right)\right], \quad v_{2}=\frac{1}{2} T_{1}^{-1} \Pi^{\prime} F_{0}^{\prime \prime} \psi^{2}, \quad v_{1 r}=T_{1}^{-1} \Pi^{\prime} F_{0}^{\prime \prime}\left(\psi, \gamma_{r}\right) .
$$

В записи $F_{0}^{\prime}$ штрих соответствует дифференцированию по $\theta$, а индекс 0 означает, что производная вычислена в точке $\left(\theta_{0}, \lambda_{0}\right)$.

Далее потребуются определители $\Delta=\operatorname{det} \mathscr{A}, \Delta_{j}=\operatorname{det} \mathscr{A}_{j}(j=1, \ldots, k+1)$. Матрицы $\mathscr{A}_{j}$ получаются заменой $j$-й строки матрицы $\mathscr{A}$ на строку $\left(-\operatorname{Re} G,-Q_{1}, \ldots,-Q_{k}\right)$, где

$$
\begin{gathered}
Q_{l}=\left(\left\langle F_{0}^{\prime \prime}\left(\psi, v_{3}\right)+F_{0}^{\prime \prime}\left(v_{4}, v_{2}\right)+F_{0 \lambda}^{\prime} v_{2}+\frac{1}{2} F_{0 \lambda}^{\prime \prime} \psi^{2}+\frac{1}{2} F_{0}^{\prime \prime \prime}\left(\psi, \psi, v_{4}\right)\right\rangle, L_{0 l}\right), \\
G=\left(F_{0}^{\prime \prime}\left(\varphi, v_{4}\right)+F_{0 \lambda}^{\prime} \varphi, \Phi\right), \quad v_{3}=T_{1}^{-1} \Pi^{\prime}\left[F_{0}^{\prime \prime}\left(\psi, v_{4}\right)+F_{0 \lambda}^{\prime} \psi\right], \quad v_{4}=T_{1}^{-1} F_{0 \lambda} .
\end{gathered}
$$

Применение метода Ляпунова-Шмидта приводит к следующей теореме.

ТЕОРема 1. Пусть для уравнения (1) с правой частью $F$, аналитической в окрестности точки $\left(\theta_{0}, \lambda_{0}\right)$, где $\left(\theta_{0}, \lambda_{0}\right)$ - равновесие: $F\left(\theta_{0}, \lambda_{0}\right)=0$, и с мультикосимметрией $\mathscr{L}$ выполнены гипотезы Н1)-Н5). Тогда справедливы следующие утвержсдения.

1) При мальх $\delta=\lambda-\lambda_{0}$ равновесия уравнения (1) в окрестности точки $\theta_{0}$ образуют семейство, аналитически зависящее от $\delta$. Точнее, существует аналитическое отображение $c: W_{0} \times\left(-\delta_{0}, \delta_{0}\right) \rightarrow H:(\beta, \delta) \rightarrow c(\beta, \delta)$ maкое, что $F\left(c(\beta, \delta), \lambda_{0}+\delta\right)=0$. Здесь $W_{0}-$ некоторая окрестность нуля пространства $\mathbb{R}^{k}$, а величина $\delta_{0}>0$. Иных равновесий при $\delta \in\left(-\delta_{0}, \delta_{0}\right)$ в $W_{0}$ нет.

2) Если вектор $F_{0}^{\prime \prime}\left(\varphi, \varphi^{*}\right)$ трансверсален подпространству $Y_{0}=\operatorname{Lin}\left\{L_{01}, \ldots, L_{0 k}\right\}$, так что нарушается хотя бы одно из равенств

$$
\left(F_{0}^{\prime \prime}\left(\varphi, \varphi^{*}\right), L_{0 i}\right)=0, \quad i=1,2, \ldots, k,
$$

то при малых $\delta$ в окрестности $\theta_{0}$ нет малых предельных чиклов. 
3) Пусть выполнены равенства (2) и неравенство $\Delta_{1} \neq 0$. Тогда $\lambda_{0}$ есть точка ответвления иикла. При этом имеются лишь две возможности: либо ответвляется единственный нормальный иикл (частота $\omega$ при $\lambda \rightarrow \lambda_{0}$ стремится $\kappa \omega_{0}$ ) и эта бифуркачия односторонняя, либо при $\lambda=\lambda_{0}$ уравнение (1) имеет однопараметрическое семейство периодических решений $\left\{\theta_{s}\right\}$, зависящее аналитически от малого параметpas $\left(\theta_{s}\right.$ не является равновесием при $\left.s \neq 0\right)$; если при этом $\delta=\lambda-\lambda_{0}$ достаточно мало, но отлично от 0 , то уравнение (1) не имеет малых периодических решений, отличных от равновесных.

4) Пусть выполняется система равенств (2) и справедливы неравенства $\Delta \neq 0$, $\Delta_{1} \neq 0$. Тогда мальй предельный чикл, стягивающийся при $\lambda \rightarrow \lambda_{0} \kappa$ равновесию $\theta_{0}$, существует, единственен, аналитически зависит от параметра $\sqrt{\delta}$ и разлагается $в$ pяд:

$$
\theta(t)=\theta_{0}+\sqrt{\varkappa_{0} \delta} \psi\left(\omega_{0} t\right)+\delta\left(\sum_{j=1}^{k} \varkappa_{j} \gamma_{j}+\varkappa_{0} v_{2}+v_{4}\right)+\cdots,
$$

где опущены члены порядка $\delta^{3 / 2}$ и выше, а $\varkappa_{l}=\Delta_{l+1} / \Delta(l=0,1, \ldots, k)$ - амплитуднье коэффичиенты. Это решение вещественно либо при малых $\delta>0$, если $\varkappa_{0}>0$, либо при $\delta<0$, когда $\varkappa_{0}<0$.

Частота $\omega$ периодического движения аналитически зависит от $\delta$ и имеет вид $\omega=$ $\omega_{0}+\mu_{1} \delta+\cdots, \mu_{1}=\operatorname{Im} G+\varkappa_{0} \operatorname{Im} g_{0}+\cdots+\varkappa_{k} \operatorname{Im} g_{k}$.

2. Метод центрального многообразия. Дополнительно к гипотезам Н1)-H5) предположим, что $\lambda$ - векторный параметр, а спектр $\sigma\left(A_{0}\right)$ лежит в замыкании левой полуплоскости.

От уравнения (1) переходим к нормализованной системе уравнений на центральном многообразии (см. [5]), в которой отбрасываем старшие слагаемые. Полученная система обладает вращательной симметрией. Ее $(k+1)$-мерная факторсистема

$$
\dot{x}=\rho\left(\varepsilon+a_{0}+A_{1} x+a \rho\right), \quad \dot{\rho}=\rho\left(\beta+\left(\beta_{1}+b_{1}\right) x+b \rho+b_{2} x^{2}\right)
$$

определена в полупространстве $\rho \geqslant 0$ пространства $(x, \rho)$. Здесь $x \in \mathbb{R}^{k}, \rho \in \mathbb{R}$ - переменные; $A_{1}: \mathbb{R}^{k} \rightarrow \mathbb{R}^{k}, \beta_{1}, b_{1}: \mathbb{R}^{k} \rightarrow \mathbb{R}$ - линейные операторы, $b_{2}: \mathbb{R}^{k} \rightarrow \mathbb{R}$ - квадратичная форма; $a_{0}, a \in$ $\mathbb{R}^{k}, b \in \mathbb{R}$ - коэффициенты, а $\varepsilon \in \mathbb{R}^{k}, \beta \in \mathbb{R}, \beta_{1}$-малые параметры.

Уравнение $\rho=0$ задает $k$-мерное семейство $\Gamma$ равновесий системы (3). Предельному циклу системы (1) соответствует равновесие $\left(x_{0}, \rho_{0}\right)$ системы $(3)$ такое, что $\rho_{0}>0$.

Бифуркация областей устойчивости. Согласно принципу линеаризации [9]-[11] равновесие $\left(x_{*}, 0\right)$ системы $(3)$ устойчиво, если его спектр, кроме точки нуль, лежит в левой полуплоскости. Устойчивость равновесия $\left(x_{*}, 0\right)$ понимается как устойчивость по Ляпунову вместе с асимптотической устойчивостью по переменной $\rho$.

Равновесия, чья устойчивость определяется линейным приближением, образуют устойчивые и неустойчивые области на гиперплоскости $\Gamma$. Если $b_{1} \neq 0$, то их общая граница деформируется гладко. Устойчивость граничных равновесий изучена в [12].

Пусть теперь $b_{1}=0$, а форма $b_{2}$ невырожденна. Это случай коразмерности 1 . Заменой переменных добиваемся выполнения в системе (3) условия $\beta_{1}=0$.

В этих условиях возможны две бифуркации.

i) Бифуркация потери устойчивости семейством равновесий. Квадратичная форма $b_{2}$ является положительно (отрицательно) определенной. Когда параметр $\beta$, возрастая, проходит через точку 0 , на устойчивом (неустойчивом) первоначально семействе равновесий возникает неустойчивая (устойчивая) окрестность точки $x=0$.

ii) Седловая бифуркация областей устойчивости. Форма $b_{2}$ знакопеременна. Когда параметр $\beta$ пересекает нулевое значение, область устойчивости или неустойчивости локально распадается на две компоненты.

Далее рассматриваются различные случаи, связанные с комбинациями вьполнения или нарушения равенств: $a_{0}=0$ - условия ответвления предельного цикла - и $b_{1}=0$, необходимого для бифуркаций i) и іi). Для каждого вида вырождения при выполнении условий типичности строится 
главная система, являющаяся упрощенной моделью для изучения локальных бифуркаций исходной системы. Она получается уменьшением числа непрерывных параметров системы (3) с помощью замен переменных, обращения времени, выбора базиса на гиперплоскости $Г$ и отбрасывания несущественных слагаемых.

1) Случай общего положения. Предельный цикл не ответвляется, и области устойчивости не бифурцируют: $a_{0} \neq 0, b_{1} \neq 0$. Главное семейство

$$
\dot{x}_{1}=\varkappa \rho, \quad \dot{x}^{k-1}=\rho\left(\xi+x_{1}\right), \quad \dot{\rho}=\rho x_{1},
$$

где $\varkappa= \pm 1, x=\left(x_{1}, x^{k-1}\right), x_{1} \in \mathbb{R}, x^{k-1}=\left(x_{2}, x_{3}, \ldots, x_{k}\right) \in \mathbb{R}^{k-1}$, имеет $k$ интегралов: $I_{1}(x, \rho)=x_{1}^{2}-2 \varkappa \rho, I_{j}(x, \rho)=2 \varkappa x_{j}-x_{1}^{2}-2 \xi_{j} x_{1}(j=2, \ldots, k)$.

2) Ответвление предельного цикла без бифуркации области устойчивости: $a_{0}=0, b_{1} \neq 0$. Рассмотрим главное семейство

$$
\dot{x}=\rho\left(A_{1} x+a \rho\right), \quad \dot{\rho}=\rho\left(\beta+b^{1} x_{1}\right)
$$

с ненулевыми коэффициентами $b^{1} \in \mathbb{R}, a \in \mathbb{R}^{k}$, мальм параметром $\beta \in \mathbb{R}$ и обратимым оператором $A_{1}$ таким, что $A_{1}^{-1} a=\left(q_{1}, \ldots, q_{k}\right), q_{1} \neq 0$.

Гиперповерхность $\beta+b^{1} x_{1}=0$ отделяет устойчивую часть равновесий семейства $Г$ от неустойчивой. Равновесие системы (4) $x_{*}=-\rho_{*} A_{1}^{-1} a, \rho_{*}=\beta /\left(b^{1} q_{1}\right)$ при $\rho_{*}>0$ соответствует циклу системы (1). Значит, точка $\beta=0$ есть точка ответвления цикла.

3) Ответвление предельного цикла в случае бифуркации областей устойчивости: $a_{0}=0$, $b_{1}=0$. Коэффициент $b \neq 0$, а линейный оператор $A_{1}$ и форма $b_{2}$ невырожденны. Заменой переменных обращаем в 0 функционал $\beta_{1}$. Изучение фазового портрета главного семейства

$$
\dot{x}=\rho\left(\varepsilon+A_{1} x\right), \quad \dot{\rho}=\rho\left(\beta+b \rho+b_{2} x^{2}\right)
$$

сводится к проведенному выше исследованию устойчивости равновесий семейства $\rho=0$ (бифуркации і) и іi) ) и рассмотрению системы

$$
\dot{x}=\varepsilon+A_{1} x, \quad \dot{y}=\beta+b y+b_{2} x^{2}
$$

в полупространстве $y>0$ пространства $(x, y)$.

Бифуркация рождения цикла при изменении параметра $(\beta, \varepsilon)$ вдоль некоторой кривой $\gamma \subset \mathbb{R}^{k+1}$ происходит, когда $\gamma$ пересекает гиперповерхность $S: \beta=-b_{2}\left(A_{1}^{-1} \varepsilon\right)^{2}$. Ответвление цикла без запаздывания (т.е. вместе с образованием области неустойчивости) имеет место только тогда, когда форма $b_{2}$ знакоопределенна, а кривая $\gamma$ пересекает $S$ в точке $(0,0)$.

\section{СПИСОК ЦИТИРОВАННОЙ ЛИТЕРАТУРЫ}

1. Юдович В. И. // ПММ. 1998. Т. 62. №1. С. 22-34. 2. Kurakin L. G., Yudovich V. I. // Chaos. 1997. V. 7. №3. Р. 376-386. 3. Куракин Л.Г., Юдович В. И. // Докл. РАН. 1998. Т. 358. №3. С. 346-349. 4. Юдович В.И. // ПММ. 1971. Т. 35. № 4. С. 638-655. 5. Арнольд В.И. // УМН. 1972. Т. 27. № 5. С. 5-220. 6. Куракин Л.Г., Юдович В.И. Бифуркация рождения цикла в динамической системе с несколькими косимметриями. Ч. 1 // Деп. ВИНИТИ. 1997. №1074-В97. 7. Куракин Л.Г., Юдович В.И. Бифуркация рождения цикла в динамической системе с несколькими косимметриями. Ч. 2 // Деп. ВИНИТИ. 1998. №150-В98. 8. Юдович В.И. // Матем. заметки. 1991. Т. 49. № 5. С. 142-148. 9. Ллпунов А. М. Общая задача об устойчивости движения. М.: Гостехиздат, 1950. 10. Малкин И.Г. Некоторые задачи теории нелинейных колебаний. М.: Гостехиздат, 1956.

11. Каменков Г. В. Избранные труды. Устойчивость и колебания нелинейных систем. Т. 2. М.: Наука, 1972. 12. Куракин Л. Г. // Матем. заметки. 1998. Т. 63. №4. С. 572-578. 